\title{
ELETROFLOCULAÇÃO, EMPREGANDO ELETRODOS DE FERRO, PARA O TRATAMENTO DE EFLUENTES LÍQUIDOS DE LATICÍNIOS
}

\author{
G. CENI ${ }^{1,2}$, R. MORES ${ }^{1}$, J. D. PRECZWSKI ${ }^{1}$, V. D. ADAM ${ }^{1}$, M. H. BOPSIN ${ }^{1}$, \\ T. L. BENAZZI ${ }^{3}$, R. M. DALLAGO ${ }^{1}$ e J. STEFFENS ${ }^{1}$
}

\begin{abstract}
${ }^{1}$ Universidade Regional Integrada do Alto Uruguai e das Missões - URI - Campus Erechim. ${ }^{2}$ IFC - Instituto Federal de Educação Ciência e Tecnologia Catarinense - Câmpus Concórdia. ${ }^{3}$ IFRS - Instituto Federal de Educação Ciência e Tecnologia do Rio Grande do Sul - Câmpus Erechim.

E-mail para contato: eaceni@hotmail.com
\end{abstract}

\begin{abstract}
RESUMO - Visando a diminuição dos impactos ambientais as indústrias estão buscadas novas alternativas para o tratamento de seus resíduos. Sendo que as indústrias de laticínios apresentam um alto consumo de água, gerando um grande número de efluentes que têm em sua composição uma elevada concentração de matéria orgânica. Dentro deste contexto, a técnica de eletrofloculação tem se mostrado eficiente na redução de substâncias em efluentes industriais com as mais variadas características. Neste estudo o objetivo foi realizar o tratamento do efluente de uma indústria de laticínio comparando a técnica de coagulação convencional com a técnica de eletrofloculação utilizando sistemas de bancada. Os ensaios foram realizados em duas etapas de tratamento, pelo método convencional, e pelo método de eletrofloculação onde foram analisados os parâmetros de remoção como cor, turbidez, $\mathrm{N}_{\text {total }}$, ST, e DQO. E realizou-se o acompanhamento do $\mathrm{pH}$. Os resultados obtidos apresentaram resultados bons de remoção, ao considerar o $\mathrm{N}_{\text {total }}$ e a DQO como parâmetros de maior relevância em relação aos resultados obtidos na condição empregando $14 \mathrm{~V}, 2,5 \mathrm{~cm}$ e 15 minutos de tratamento, obteve-se remoções de Cor, Turbidez, $\mathrm{N}_{\text {total }}$, DQO e ST de 93,3\%, $97,1 \%, 72,8 \%$ e $52,6 \%$ respectivamente, ou seja, maior DE e maior T maior a eficiência de tratamento por eletrofloculação.
\end{abstract}

\section{INTRODUÇÃO}

O desenvolvimento urbano, industrial e agrícola tem como um dos principais fatores limitantes a disponibilidade hídrica. A disputa pela água e a escassez hídrica gera conflitos de usos e impõe restrições no consumo, mobilizando os diversos setores da sociedade na tentativa de garantir uma relação mais harmônica entre as suas atividades e os recursos hídricos.

Em função das características específicas de cada processo industrial, tem-se o consumo maior ou menor de água e por consequência a variação do volume de efluentes líquidos gerados. Estes quando não tratados adequadamente, possuirão um alto potencial de contaminação da água, solos e ar. Para evitar a degradação do meio ambiente, a legislação 
ambiental vem cada vez mais obrigar e cobrar dos empreendimentos poluidores a adoção de soluções para os problemas ambientais gerados. Atualmente diversas formas vêm sendo adotadas para obrigar os mais variados tipos de empreendimentos a se adequarem às exigências ambientais. As mais comumente utilizadas são: pesadas multas, compensações ambientais de área degradadas ou em outras áreas e algumas vezes podendo chegar a paralisação da atividade industrial até a adequação às exigências dos órgãos ambientais.

Em consonância com as crescentes tendências mundiais de preservação do meio ambiente, desenvolvimento de novas tecnologias de remediação ambiental e aprimoramento das já existentes, é que a eletrofloculação tem sido reavaliada como uma alternativa promissora no tratamento de diversos tipos de efluentes (ABUZAID, et al., 1999; AVSAR, et al., 2008, ILHAN, et al., 2008), uma vez que a mesma oferece uma alta eficiência de remoção do processo (GUVEN et al., 2009). O processo de eletrofloculação proporciona um ambiente físico/químico que permite a desestabilização do poluente pela oxidação eletrolítica e sua coagulação, adsorção, precipitação e flutuação subsequentes, evitando a introdução de outro agente coagulante (MOLLAH et al., 2004; ADHOUM N., 2004).

Portanto, o objetivo deste trabalho foi comparar o processo de tratamento de coagulação convencional e o processo de eletrofloculação para o tratamento de efluentes líquidos de laticínios,

\section{MATERIAIS E MÉTODOS}

\subsection{Efluente}

Para os experimentos um efluente sintético de leite em pó (solução de $2 \mathrm{~g} / \mathrm{L}$ de leite em pó) (LEAL et al., 2006) foi preparado e caracterizado físico-quimicamente quanto a: cor, turbidez, demanda química de oxigênio (DQO), condutividade e $\mathrm{pH}$. O pH do efluente foi ajustado, adicionando-se uma solução de $\mathrm{NaOH}$ ou $\mathrm{HCl} 0,1$ mol.L-1 (Vetec) para os experimentos de coagulação e eletrofloculação.

\subsection{Etapa I: Coagulação convencional}

$\mathrm{Na}$ etapa de coagulação convencional utilizou-se 2L de efluente. Para os ensaios de coagulação, foi avaliada o efeito da quantidade de $\mathrm{FeCl}_{3}$ adicionada $(4,0 ; 4,5 ; 5,0 ; 5,5 ; 6,0$; 6,5 e 7,0 mL), a partir de uma solução a $10 \%$ da mesma. Após a adição do agente coagulante, a solução ficou sob agitação de $2 \mathrm{~min}$, sendo posteriormente transferida para um cone Imhoff para análise de sólidos sedimentáveis, anotando-se o valor do material sedimentado (em mililitros) e analisaram-se os seguintes parâmetros: cor, turbidez, carbono orgânico total (COT), demanda química de oxigênio (DQO), Nitrogênio Total $\left(\mathrm{N}_{\text {total }}\right)$, condutividade e $\mathrm{pH}$. Os experimentos foram conduzidos em triplicata e as análises em duplicata.

\subsection{Etapa II: Tratamento por Eletrofloculação}

$\mathrm{O}$ reator de EF em batelada possui-a capacidade de tratar 1,7 litros de efluente, com dois pares de eletrodos de ferro, com dimensões de $130 \mathrm{~mm}$ de altura e $70 \mathrm{~mm}$ de largura, ligados a uma fonte de corrente contínua (MIT DC POWER SUPPLY MS 3005), sendo o sistema 
mantido sob agitação constante (LOGEN scientific). O pH do efluente foi ajustado para 6,0 com uma solução de ácido sulfúrico $1 \mathrm{~mol} / \mathrm{L}$ (Vetec).

Os experimentos foram realizados para identificar a condição operacional que influencia no desempenho processo de eletrofloculação para remoção dos contaminantes das águas residuárias de laticínios. Foram avaliados os parâmetros Voltagem (V), Distância entre os eletrodos (DE), Tempo (T).

O desempenho dos processos foi avaliado por meio das seguintes parâmetros: cor e a turbidez foram mensuradas através do método espectrofotométrico, utilizando o colorímetro o Hach (DR870). O pH foi medido usando o pHmetro da Metrhm (pH Lab 837). Os níveis de COT foram analisados segundo a metodologia padrão ISO-1987 em equipamento Shimadzu (TOC-5000A). A DQO foi determinada usando o método colorimétrico em microescala (5220D, APHA, 1992), onde as amostras foram preparadas e conduzidas para digestão em um termoreator Marconi (Dry Block MA 4004), após a digestão realizou-se as leituras no colorímetro digital Hach (DR870).

\section{RESULTADOS E DISCUSSÕES}

\subsection{Coagulação: Método Convencional}

Os resultados da caracterização para as demais condições de agente coagulante são encontrados na Tabela 1 .

Tabela 1 - Resultados dos parâmetros analisados

\begin{tabular}{|c|c|c|c|c|c|c|c|c|c|c|}
\hline \multirow{3}{*}{$\begin{array}{c}\text { Dosagem de } \\
\text { Coagulante (mL) }\end{array}$} & \multicolumn{10}{|c|}{ Parâmetros Analisados } \\
\hline & \multicolumn{2}{|c|}{$\begin{array}{c}\text { Cor } \\
\text { REMOÇÃO }\end{array}$} & \multicolumn{2}{|c|}{$\begin{array}{c}\text { Turbidez } \\
\text { REMOÇÃO }\end{array}$} & \multicolumn{2}{|c|}{$\begin{array}{c}\text { DQO } \\
\text { REMOÇÃO }\end{array}$} & \multicolumn{2}{|c|}{$\begin{array}{c}\text { ST } \\
\text { REMOÇÃO }\end{array}$} & \multicolumn{2}{|c|}{ pH } \\
\hline & PtmgL $^{-1}$ & $\%$ & NTU** & $\%$ & $\mathrm{O}_{2} \mathrm{mgL}^{-1}$ & $\%$ & $\mathrm{mgL}^{-1}$ & $\%$ & Inicial & Final \\
\hline Efluente Bruto & 9541,67 & $\mathbf{0}$ & 1217,97 & $\mathbf{0}$ & 2884,23 & $\mathbf{0}$ & 0,080 & - & - & - \\
\hline 4,0 & 325,00 & 96,59 & 45,83 & 96,24 & 1356,25 & 52,98 & 0,043 & 46,06 & 5,91 & 5,25 \\
\hline 4,5 & 287,50 & 96,99 & 41,63 & 96,58 & 982,50 & 65,94 & 0,049 & 38,69 & 6,04 & 5,32 \\
\hline 5,0 & 239,58 & 97,49 & 31,25 & 97,43 & 1737,50 & 36,51 & 0,052 & 34,58 & 6,05 & 4,96 \\
\hline 5,5 & 93,75 & 99,02 & 25,00 & 97,94 & 2162,50 & 25,02 & 0,043 & 46,81 & 6,05 & 4,80 \\
\hline 6,0 & 212,50 & 97,77 & 37,50 & 96,92 & 1662,50 & 42,36 & 0,049 & 39,12 & 5,93 & 4,44 \\
\hline 6,5 & 264,58 & 97,23 & 40,63 & 96,66 & 1391,67 & 51,75 & 0,046 & 42,46 & 6,08 & 4,70 \\
\hline 7,0 & 590,63 & 92,81 & 95,83 & 92,13 & 1475,00 & 48,86 & 0,049 & 38,28 & 5,92 & 4,12 \\
\hline
\end{tabular}


Pode-se observar na Tabela 1 uma variação distinta quando comparado os resultados de pH para os demais parâmetros. $\mathrm{O}$ pH final apresentou uma tendência de redução quando adicionado maior quantidade de $\mathrm{FeCl}_{3}$ devido as características do agente coagulante, por ser um sal ácido. Quanto a porcentagem de remoção dos parâmetros dos experimentos feitos com 4,$0 ; 4,5 ; 5,0 ; 5,5 ; 6,0 ; 6,5$ e 7,0 $\mathrm{mL}$ respectivamente, houve um aumento significativo da remoção da cor e turbidez a partir de $4,0 \mathrm{~mL}$ até na faixa dos $5,5 \mathrm{~mL}$. Tendo como melhor resultado para cor e turbidez, a dosagem de $5,5 \mathrm{~mL}$ de agente coagulante, chegando a 99,02\% de remoção de Cor e 97,94\% de Turbidez, ambos os parâmetros apresentando similaridade, mostrando-se um resultado extremamente positivo. A cima desta faixa de dosagem do $\mathrm{FeCl}_{3}$, a tendência é a redução da eficiência do processo de coagulação, sendo a dosagem em excesso, não induz a sua agregação. Os valores de eficiência na redução de DQO foram de $65,94 \%$ com $4,5 \mathrm{~mL}$ de agente coagulante destaca-se como melhor resultado no teste convencional.

Os sólidos totais, os resultados de remoção oscilaram entre 34 e $46 \%$ de remoção. Em relação à sedimentação do efluente, a mesma ocorreu de forma lenta e concisa, de modo que a parte poluente do efluente ocupou a parte inferior do cone Imhoff conforme mostra a Figura 1.

Figura 1 - Análise visual do efluente sintético bruto e tratado, utilizando 5,5 mL de agente coagulante, após 60 minutos de decantação.
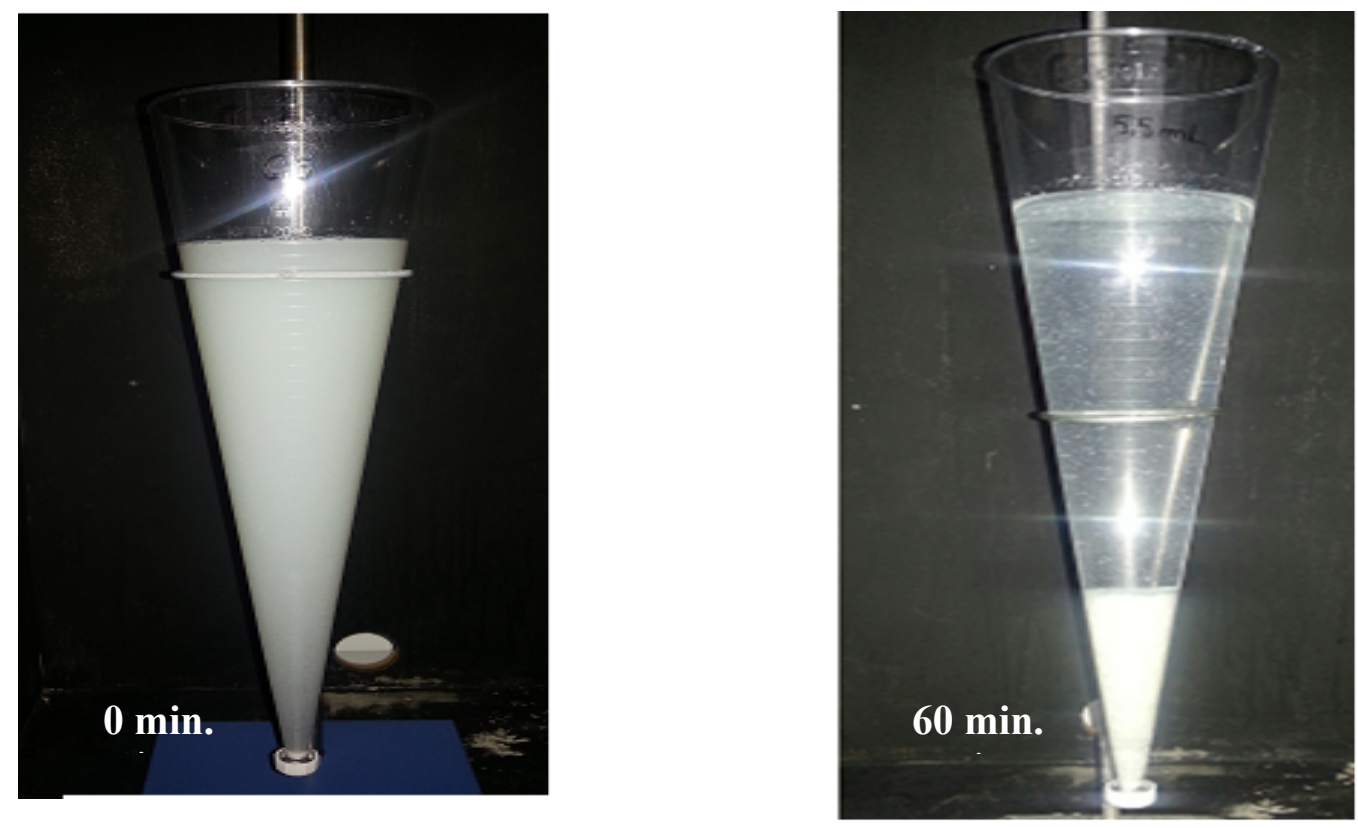

Os flocos formados para ambos os experimentos, são relativamente finos, com o efluente apresentando uma redução no $\mathrm{pH}$ final, devido as características do agente coagulante. Todas as condições de dosagens utilizadas (entre 4 e $7 \mathrm{~mL}$ ), apresentaram boa separação das fases líquida e sólida. 


\subsection{Coagulação: Processo Eletroquímico}

Após os testes realizados com o método de coagulação convencional foram realizado os ensaios utilizando o processo de eletrofloculação com obtenção dos resultados mostrados na tabela 2, como as eficiências de remoção operacionais para as variáveis dependentes.

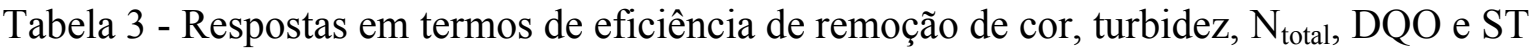

\begin{tabular}{cccc|cccccc}
\hline & \multicolumn{3}{c|}{ Condições Operacionais } & \multicolumn{5}{c}{ Eficiência de Remoção (\%) } \\
\cline { 2 - 9 } Ensaios & $\mathbf{V}^{*}(\mathbf{V})$ & $\mathbf{D E *}(\mathbf{c m})$ & $\mathbf{T}^{*}(\mathbf{m i n})$. & COR & TURBIDEZ & $\mathbf{N}_{\text {total }}$ & DQO & ST & pH \\
\hline 1 & $-1(14)$ & $-1(1,5)$ & $-1(5)$ & 55,64 & 60,56 & 77,85 & 20,55 & 27,02 & 8,40 \\
2 & $-1(14)$ & $-1(1,5)$ & $1(15)$ & 82,92 & 91,80 & 89,97 & 60,35 & 49,83 & 11,24 \\
3 & $-1(14)$ & $1(2,5)$ & $-1(5)$ & 73,19 & 82,79 & 59,03 & 57,18 & 37,56 & 8,10 \\
4 & $-1(14)$ & $1(2,5)$ & $1(15)$ & 93,27 & 97,09 & 91,26 & 72,80 & 52,06 & 11,10 \\
5 & $1(22)$ & $-1(1,5)$ & $-1(5)$ & 95,10 & 100,00 & 61,68 & 80,98 & 57,12 & 9,30 \\
6 & $1(22)$ & $-1(1,5)$ & $1(15)$ & 92,62 & 96,49 & 84,12 & 73,02 & 39,24 & 11,07 \\
7 & $1(22)$ & $1(2,5)$ & $-1(5)$ & 94,53 & 96,15 & 86,38 & 71,94 & 45,71 & 9,70 \\
8 & $1(22)$ & $1(2,5)$ & $1(15)$ & 91,87 & 95,45 & 91,50 & 70,80 & 46,14 & 10,95 \\
\hline
\end{tabular}

* $\mathrm{V}=$ Voltagem; $\mathrm{DE}=$ Distância entre os eletrodos; $\mathrm{T}=$ Tempo reacional.

A eficiência da remoção de cor, turbidez e DQO apresentou os melhores resultados $95,10 \%$ e $100 \%$ 80,98\% no ensaio 5 quando empregado uma condição de $22 \mathrm{~V}, 1,5 \mathrm{~cm}$ e 5 minutos de tratamento mas portanto apresentou valores de remoção menores para $\mathrm{N}_{\text {total }}$ de $61,68 \%$ respectivamente, mas mesmo assim obteve valor maior que $50 \%$ de remoção , ou seja, maior voltagem $\mathrm{V}$, menor $\mathrm{DE}$ e menor $\mathrm{T}$ maior a eficiência de tratamento por eletrofloculação.

Nas condições avaliadas em relação ao parâmetro pH observou-se um aumento com o tempo reacional (Tabela 3). Os pHs finais variaram entre 8,40 e 11,24. Os experimentos conduzidos empregando as maiores $\mathrm{V}$ e/ou $\mathrm{T}$ apresentaram um aumento mais pronunciado. Esse aumento de $\mathrm{pH}$ está relacionado com a produção de íons $\mathrm{OH}^{-}$, que no caso de eletrodos de ferro ocorre a formação de $\mathrm{Fe}(\mathrm{OH})_{2}$ e, ou, $\mathrm{Fe}(\mathrm{OH})_{3}$.

$\mathrm{O}$ aumento no $\mathrm{pH}$ pode estar vinculado a produção de hidrogênio molecular $\left(\mathrm{H}_{2}\right)$ no cátodo, a qual ocorre pela redução dos íons $\mathrm{H}^{+}{ }_{(\mathrm{aq})}$. Outras reações participantes e que influenciam no equilíbrio são: hidrólise do $\mathrm{Fe}$, formação de oxigênio no ânodo, liberação de $\mathrm{CO}_{2}$ (pelo borbulhamento de hidrogênio no cátodo) e formação de outros hidróxidos insolúveis (CRESPILHO e REZENDE, 2004).

\section{CONCLUSÃO}

Para o teste de coagulação convencional os melhores resultados foram apresentados com as dosagens de 4,5 mL apresentando, 65,94\% de remoção para a DQO, e com 5,5 mL de 


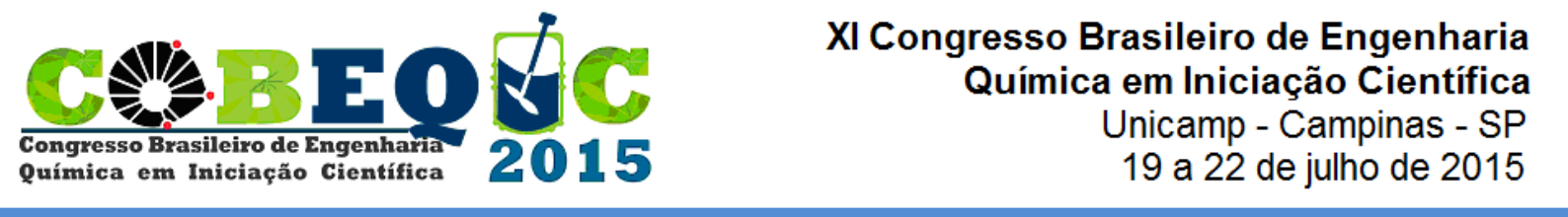

agente coagulante apresentando $99 \%$ de remoção para Cor e 97,94\% para Turbidez, mostrando que as diferentes dosagens utilizadas para os experimentos influenciaram no processo de coagulação convencional.

Para os ensaios utilizando o método de eletrofloculação, apresentaram bons resultados quanto aos parâmetros estudados. Podemos destacar o parâmetro de $22 \mathrm{~V}, \mathrm{DE} 1,5 \mathrm{~cm}$ e $5 \mathrm{~min}$, apresentando remoções de $80,98 \%$ de DQO e chagando aos $100 \%$ de remoção para turbidez. Mostrando-se assim uma técnica rápida e de grande eficiência para tratamento de efluentes e água residuárias com altas taxas de poluentes.

\section{REFERÊNCIAS}

ABUZAID N.S., AL-HAMOUZ Z., BUKHARI A.A., ESSA M.H., Electrochemical treatment of nitrite using stainless steel electrodes, Water Air Soil Pollut. 109 (1999) 429-442.

ADHOUM N., MONSER L., 2004. Decolourization and removal of phenolic compounds from olive mill wastewater by electrocoagulation[J]. Chem Eng Process, 43(10): 12811287.

AVSAR Y. KURT U., GONULLU T.. Comparison of classical chemical and electrochemical processes for treating rose processing wastewater. Journal of Hazardous Materials 148 (2007) 340-345.

GUVEN G., PERENDECI A., TANYOLA A., Electrochemical treatment of simulated beet sugar factory wastewater, Chem. Eng. J. 151 (2009) 149-159.

ILHAN F., KURT U., APAYDIN O., GONULLU M.T., Treatment of leachate by electrocoagulation using aluminum and iron electrodes, J. Hazard. Mater. 154 (2008) 381-389.

LEAL M.C.M.R., FREIRE D.M.G., CAMMAROTA M.C., SANT'ANNA JR. G.L., Effect of enzymatic hydrolysis on anaerobic treatment of dairy wastewater. Process Biochemistry, v. 41, 2006.

MOLLAH, M. Y. A., Schennach, R., Parga, J. R. and Cocke, D. L. (2001) Electrocoagulation (EC) - Science and applications. Journal of Hazardous Materials, B84: 29 - 41. 